\title{
Antibiotic resistance and integrons in Shiga toxin-producing Escherichia coli (STEC)
}

\author{
Rocío Colello $^{1}$, Analía I. Etcheverría ${ }^{1}$, Jose A. Di Conza ${ }^{2}$, \\ Gabriel O. Gutkind ${ }^{2}$, Nora L. Padola ${ }^{1}$ \\ ${ }^{1}$ Laboratorio de Inmunoquímica y Biotecnología, Facultad de Ciencias Veterinarias, \\ Centro de Investigación Veterinaria de Tandil, Consejo Nacional the Investigaciones Científicas y \\ Técnicas, Universidad Nacional del Centro de la Provincia de Buenos Aires, Buenos Aires, Argentina. \\ ${ }^{2}$ Laboratorio de Resistencia Microbiana, Cátedra de Microbiología, Facultad de Farmacia y Bioquímica, \\ Universidad de Buenos Aires, Buenos Aires, Argentina.
}

Submitted: June 23, 2013; Approved: June 6, 2014.

\begin{abstract}
Shiga toxin-producing Escherichia coli (STEC) cause hemorrhagic colitis (HC) and hemolyticuremic syndrome in humans (HUS). Cattle are the main reservoir of STEC and transmission to humans occurs through contaminated food and water. Antibiotics are used in pig production systems to combat disease and improve productivity and play a key role in the dissemination of antibiotic resistance genes to the bacteria. Integrons have been identified in resistant bacteria allowing for the acquisition and dissemination of antibiotic resistance genes. STEC strains isolated from humans and animals have developed antibiotic resistance. In our laboratory, 21 non-157 STEC strains isolated from pigs were analyzed to detect class 1 and 2 integrons by PCR. Eight carried integrons, 7 of them harbored intl2. In another study 545 STEC strains were also analyzed for the presence of intll and intl2. Strains carrying intl 1 belonged to isolates from environment $(\mathrm{n}=1)$, chicken hamburger $(\mathrm{n}=2)$, dairy calves $(\mathrm{n}=4)$ and pigs $(\mathrm{n}=8)$. Two strains isolated from pigs harbored intl2 and only one intll/intl2, highlighting the presence of intl2 in pigs. The selection for multiresistant strains may contribute to the emergence of antibiotic resistant pathogens and facilitate the spreading of the mobile resistance elements to other bacteria.
\end{abstract}

Key words: STEC, integrons, multiresistance, antibiotics, public health.

\section{Shiga Toxin-Producing Escherichia coli (STEC)}

STEC have been an important cause of foodborne illnesses worldwide that can cause gastrointestinal disease in humans, particularly infections that result in lifethreatening complications such as hemolytic-uremic syndrome (HUS) and hemorrhagic colitis (HC) (Paton and Paton, 1998). HUS is a syndrome characterized for renal failure, hemolytic anemia and thrombocytopenia that affects children less than 5 years old (Voyer, 1996). This damage is produced by the action of cytotoxins: Stx 1 and Stx2, encoded by stx 1 and stx 2 genes, respectively. The strains often carry the eae gene, encoding intimin an adherence membrane protein which is required for attachment to the host intestinal mucosa (Kaper et al., 1994). These strains produce putative accessory virulence such as a plasmid-encoded enterohemolysin $(e h x A), \quad$ a catalase-peroxidase (katP), an autoagglutinating adhesion (Saa), a subtilase cytotoxin (subAB), among others (Adu-Bovie et al., 1998; Etcheverría and Padola, 2013).

\section{Transmission of STEC to Humans}

Transmission to people occurs primarily via ingestion of inadequately processed contaminated food or water and less frequently through contact with infected manure, animals and people (Hussein and Sakuma, 2005). Cattle are the main reservoir of STEC (Thomas et al., 2012) but sheep are also considered a significant source of infection in humans (La Ragione et al., 2009). Furthermore, STEC has

Send correspondence to R Colello. Laboratorio de Inmunoquímica y Biotecnología, Facultad de Ciencias Veterinarias, Consejo Nacional the Investigaciones Científicas y Técnicas, Universidad Nacional del Centro de la Provincia de Buenos Aires, Buenos Aires, Argentina. E-mail: rocioc@vet.unicen.edu.ar. 
been isolated from goats, horses, dogs, rabbits and seagulls (Naylor et al., 2005; Wetzel and LeJeune, 2006; Jay et al., 2007). In addition, pigs are also involved in the transmission of STEC in several countries and some HUS outbreaks have been associated with the consumption of food products derived from pigs such as dry sausages (Notario et al., 2000; Parma et al., 2000).

\section{Antibiotic Resistance: Integrons}

Antibiotic resistance is recognized as a global problem in human and veterinary medicine. For more than 50 years, antibiotic agents have been an essential component of the infectious disease treatment, and the use of these has resulted in the development and spread of antibiotic multiresistance. Magiorakos et al. (2011) multidrug-resistant strain when bacteria are resistant to three or more antibiotic classes. In this work the term multiresistance is used as a synonymous of multidrug-resistant. Any kind of antibiotic used, be it for human, animal or plant health purposes can select for the emergence of resistance and further promote the dissemination of resistant bacteria and resistance genes (Newell et al., 2010; Bush et al., 2011). Bacteria are able to acquire antibiotic resistance genes that provide protection against most antibiotics. The dissemination and acquisition of such genes by horizontal gene transfer has led to the rapid emergence of antibiotic resistance among bacteria (Carattoli, 2013). A system described at the end of the 1980s has been referred to as bacterial integrons identified in multiple resistant bacteria that play an important role in the acquisition and dissemination of antibiotic resistance genes. Integrons are elements that contain the genetic determinants of the components of a site-specific recombination system that recognizes and captures the mobile genes cassette. The essential component of an integron is based on a functional platform (also called 5 conserved segment, $5 \mathrm{CS})$, composed of three key elements: the intl gene encoding a site-specific recombinase, an adjacent site, att, that is recognized by the integrase and is the receptor site for the cassettes encoded gene and a promoter suitably oriented for the expression of the cassette (Hall and Collis, 1995). Integrons are not able to self-transfer, but can associate themselves with insertion sequences present in tranposons and (or) conjugative plasmids that serve as a vehicle for their intra or inter-species transmission (Fluit and Schmitz, 1999; Waldor, 2010). It is known that the presence of integrase is potentially indicative of strains capable of recruiting antibiotic resistance genes (Di Conza and Gutkind, 2010).

Based on the aminoacid sequence of the Intl protein, five classes of integrons are known to play a role in the dissemination of antibiotic resistance genes (Mazel, 2006; Cambray et al., 2010). Classes 1 and 2 are the most prevalent in clinical isolates and are largely implicated in the dissemination of antibiotic resistance and have also been the focus of numerous widespread studies (Ploy et al., 2000a).
Class 1 integrons represents the most common structure and is characterized by the presence of two conserved segments, separated by a segment of variable length (sequence which includes inserted antibiotic resistance gene cassettes). The 5' -CS contains the intI gene, the attI site and the promoter, while the 3'-conserved segment (3'-CS) commonly codes for the sull gene, conferring resistance to sulphonamides and the $q a c E \Delta l$ gene, conferring resistance to quaternary ammonium compounds used as disinfectants (Stokes and Hall, 1989).

Class 2 integrons are embedded within transponibles elements $\operatorname{Tn} 7$ and its derivatives. The gene coding for the integrase class 2 (intl2) is located at the 5-CS (Hansson et al., 2002). Often the gene intl2 is interrupted prematurely by a codon stop, and this make it a pseudogene, which would explain the few arrangements that are described in the databases, compared to the number of integrons class 1 (Recchia and Hall, 1995). Its 3 conserved segment usually contains five ths genes involved in the movements of the transposon (tns $A$, tns $B$, tns $C$, tnsD, tnsE) (Ploy et al., 2000b).

\section{STEC, Antibiotic Resistance and Integrons}

Some reports indicate that STEC strains isolated from humans and animals have acquired antibiotic resistance genes almost twenty years ago (Farina et al., 1996; Zhao et al., 2001). Class 1 and 2 integrons are strongly associated with multiresistance to antibiotic in STEC isolated from different origins in the world (Guerra et al., 2006; Vali et al., 2007). On May 2011 in Germany, a large outbreak of HUS associated with the rare hybrid strain of Shiga toxinproducing Escherichia coli (STEC) and Escherichia coli enteroaggregative (EAEC) serotype O104:H4 occurred (Askar et al., 2011). There are important differences between this outbreak and previous outbreaks of STEC infection as the majority of the cases of the HUS (90\%) occurred in adults rather than in children, with the majority occurring in women. A total of 3816 cases (including 54 deaths) were reported, 845 of which (22\%) involved HUS (Frank et al., 2011). All isolates classified as the outbreak strain were resistant to all penicillins and cephalosporins and susceptible to carbapenems. This resistance pattern is typical for CTXM-15 $\beta$-lactamases (Bielaszewska et al., 2011). CTX-Mtype enzymes were also described in other STEC serotype O111:H8 (Valat et al., 2012) and O26:H11 (Ishii et al., 2005).

Vali et al. (2007) tested 297 STEC O26, 152 STEC O103 and 13 STEC O145 isolated in two cohorts of Scottish beef cattle from two farms. Isolates were screened for the presence of class 1 and 2 integrons. E. coli $\mathrm{O} 26$ isolates were susceptible to apramycin, cefalexin, chloramphenicol and nalidixic acid. E. coli $\mathrm{O} 103$ isolates were susceptible to apramycin, nalidixic acid and neomycin. E. coli $\mathrm{O} 145$ isolates were susceptible to apramycin, cephalexin, chloram- 
phenicol and nalidixic acid. Among STEC O26, O103 and O145 there were 4, 4 and 1 isolates, respectively, that contained class 1 integrons. Class 2 integrons were detected in only one $\mathrm{O} 145$ isolate.

Over a period of time between 1983 and 2003, a total of 105 epidemiologically unrelated STEC O111 isolates from humans and cattle from Germany were tested for susceptibility to 17 antibiotics agents. Resistant isolates were screened by molecular methods for class 1 and 2 integrons. Resistance was found in $76 \%$ of the isolates, with a prevalence of $72 \%$ for multiresistance. The most prevalent resistance patterns were to streptomycin, sulfamethoxazole and tetracycline (72-68\%), followed by spectinomycin, ampicillin and kanamycin/neomycin (39-25\%). Class 1 integrons were detected in $41 \%$ of the isolates while class 2 integrons were found in only one (Guerra et al., 2006).

In the USA 93 (34\%) of 274 STEC from poultry, cattle, swine, and humans were resistant to streptomycin, 89 $(32 \%)$ to sulfamethoxazole, $83(30 \%)$ to tetracycline, 48 $(18 \%)$ to ampicillin, $29(11 \%)$ to cefalothin, $22(8 \%)$ to trimethoprim/sulfamethoxazole, $18(7 \%)$ to gentamicin, 13 (5\%) to chloramphenicol, and $10(4 \%)$ to cefoxitin. Fortythree $(16 \%)$ of the STEC isolates harbored class 1 integrons and $41(95 \%)$ of STEC positive to class 1 integrons were resistant to one or more antibiotics (Sing et al., 2005).

Another study was conducted in USA during 2005 on 24 E. coli isolates from dairy farms. From that, 14 E. coli were isolated from dairy cows with mastitis (ECDM), 9 STEC 0157:H7 from dairy cow and only one from bulk tank milk. These strains were evaluated for sensitivity to 19 antibiotics used in human and/or veterinary medicine. Class 1 integrons were found only in eight of 10 isolates (one STEC 0157:H7 and seven ECDM). Eight of 10 STEC O157:H7 and six of 14 ECDM were susceptible to all tested antibiotics (Murinda et al., 2005).

In another research, antibiotic resistance patterns and molecular characteristics were determined in 32 STEC strains previously identified in São Paulo State, Brazil associated with 21 human infections and 11 cattle feces. The highest resistance was identified for tetracycline (100\%), streptomycin (78\%) and trimethoprim-sulfamethoxazole (56\%). Eleven STEC strains showed resistance to ampicillin. The class 1 integrase gene (intII) was detected in seven $(22 \%)$ strains, and five from seven (5/7) belonged to the O111:H8 serotype (Cergole-Novella et al., 2011).

In other studies, a total of 50 isolates of STEC, including 29 O157:H7 and 21 non-O157 STEC strains were analyzed for antibiotic susceptibilities and the presence of class 1 integrons. Seventy-eight percent of the isolates exhibited resistance to two or more antibiotic classes. Multiresistance to streptomycin, sulfamethoxazole, and tetracycline was most often observed. Class 1 integrons were identified among nine STEC isolates, including serotypes O157:H7, O111:H11, O111:H8, O111:NM, O103:H2, O45:H2, O26:H11, and O5:NM (Zhao et al., 2001).

Two different studies were carried out in our laboratory with isolates collected in Argentina. On the other hand, a total of 21 non-O157 STEC strains isolated from pig feces recovered from ten farms (named A to J) were analyzed to detect the 5 '-conserved segment of integrons (called intII and intI2) by PCR. Eight (38\%) belonging to 4 different farms carried integrons. As a matter of interest, 7 of these isolates harbored intl2 unlike with the studies cited above. The results of PCR and resistance phenotypes are summarized in Table 1.

Furthermore, a collection of 545 STEC strains from different origins were analyzed for the presence of intll and intl2. The STEC strains carrying intll belonged to isolates from environment $(n=1)$, chicken hamburger $(n=2)$, dairy calves $(n=4)$ and pigs $(n=8)$. Moreover, two strains isolated from pigs harbored intl2 and another strain both intll/intl2. Both local studies highlighted the presence of class 2 integrons in STEC isolated from pig samples (data not published).

The antibiotics mentioned above are used commonly in pigs production system to treat and prevent diseases. Antibiotics are often used in animal production with little or no veterinary consultation making it difficult to determine which drugs are used in what quantities (McEwen and Fedorka-Cray, 2002).

Table 1 - Results of PCR and resistance phenotypes.

\begin{tabular}{lccl}
\hline Farm & Toxin subtype & 5'-CS & Antibiotic resistance \\
\hline A & $s t x 2 e$ & $i n t l 1$ & S-AMP-TET-CMP-TMS-DOX-FLOR \\
B & $s t x 2 e$ & $i n t l 2$ & S-TET-CMP-TMS-DOX-FLOR \\
B & $s t x 2 e$ & $i n t l 2$ & S-TET-CMP-DOX-FLOR \\
B & $s t x 2 e$ & $i n t l 2$ & TET-DOX-FLOR-CIP-NAL \\
B & $s t x 2 e$ & $i n t l 2$ & S-TET-CMP-TMP/SMX-DOX-FLOR \\
C & $s t x 2$ & $i n t l 2$ & S-TET-TMP/SMX-DOX-FLOR \\
C & $s t x 2$ & $i n t l 2$ & S-TET-TMP/SMX-DOX-NAL \\
D & $s t x 1$ & $i n t l 2$ & S-TET-DOX-NAL \\
\hline
\end{tabular}

CIP: Ciprofloxacin. AMP: Ampicillin. TMP: Trimethoprim/Sulfamethaxazole. DOX: Doxycyline. CMP: Chloramphenicol. FLOR: Florfenicol. TET: Tetracycline. NAL: Nalidixic acid. S: Streptomycin (Colello et al., 2012). 
Taking into account the use of antibiotics in different stages of the pig production and the increase in the consumption of pig meat during the last years, it is of high interest the knowledge about the presence of STEC strains harboring integrons in this system of animal production. Only in Argentina, according to data obtained from the National Agricultural Trade Control (ONCAA) the pig production in 2009 was estimated at 287,121 tons, $4.9 \%$ more compare to the same period in 2008 , showing the consumption of meat from pig continues increasing.

Thereby, the presence of STEC harboring integrons is of high significance because of the possibility of entrance into the food chain. Entrance into the food production implies a risk to the public health because the possibility of acquisition and transmission of the new genes conferring antibiotic resistance intra or inter species. Vignoli et al. (2005) have emphasized the importance of studying antibiotic multiresistance in pathogenic E. coli, such as STEC, from an epidemiological standpoint.

\section{Concluding Remark}

The emergence and dissemination of antibiotic resistance among STEC isolates may have potentially negative implications and the characterization of resistance mechanisms provides additional information about the epidemiology of the resistance.

Even though the antibiotic resistance itself is not a virulence factor, it has been proven that acts as a key factor in the development of the infection, therefor may be taken as a virulence like factor in specific ecological niches that colud be colonized by antibiotic resistant bacteria (Beceiro et al., 2013).

Although the degree of knowledge about integrons has changed in recent years and the information available today is extensive, there are still important points to understand and investigate. Many microorganisms carrying integrons can change and adapt quickly to different environmental niches and remain stable in several microbial lineages highlights not only its adaptive value, but also the potential for selection and dissemination of multiresistant bacteria and it is known that antibiotic resistant bacteria will increase a lot.

Even if hospitals were traditionally considered to be the major selective environment of antibiotic resistant bacteria and resistance genes, it is becoming clear that other reservoirs of resistance genes could exist (Avgustin, 2012).

Antibiotics are used in pig production systems to combat disease and improve animal productivity. Feedbased antibiotics consistently benefit productivity, increasing the ability of farms to maintain profitable margins (Cromwell et al., 1996).

Although antibiotic therapy is generally not recommended for treatment of STEC infections in humans, the indirect selection for multiresistant strains contribute to the increase of emerging antibiotic resistant pathogens and fa- cilitate the spread of these mobile resistance elements to other bacteria. Corogeanu et al. (2012) proposed that there is a chance for antibiotic treatment of patients in outbreaks of STEC with antibiotics that do not improve the release of Stx. However, an increased surveillance and the development of adequate prevention strategies are needed for public health reasons.

\section{References}

Adu-Bovie J, Frankel G, Bain C et al. (1998) Detection of intimin alpha, beta, gamma, and delta, for intimin derivatives expressed by attaching and effacing microbial pathogens. J Clin Microbiol 36:662-668.

Askar M, Faber MS, Frank C et al. (2011) Update on the ongoing outbreak of haemolytic uraemic syndrome due to Shiga toxin-producing Escherichia coli (STEC) serotype O104, Germany, May 2011. Euro Surveill 16:1-3.

Avgustin J (2012) Animal production systems as a selective environment for antibiotic resistance genes. Acta Agriculturae Slovenica 1:7-17.

Beceiro A, Tomás M, Bou G (2013) Antimicrobial resistance and virulence: a successful or deleterious association in the bacterial world? Clin Microbiol Rev 2:185-208.

Bielaszewska M, Mellmann A, Zhang W et al. (2011) Characterisation of the Escherichia coli strain associated with an outbreak of haemolytic uraemic syndrome in Germany, 2011 a microbiological study. Lancet Infect Dis 11:671-676.

Bush K, Courvalin P, Dantas G (2011) Tackling antibiotic resistance. Nature Microbiol Rev 9:894-896.

Cambray G, Guerout AM, Mazel D (2010) Integrons. Annu Rev Genet 44:141-166.

Carattoli A (2013) Plasmids and the spread of resistance. Int J of Med Microbiol 303:298-304.

Cergole-Novella MC, Pignatari AC, Castanheira M et al. (2011) Molecular typing of antimicrobial-resistant Shiga-toxinproducing Escherichia coli strains (STEC) in Brazil. Res Microbiol 162:117-123.

Colello R, Moredo F, Etcheverría A et al. (2012) Detection of integrons class 1 and class 2 in VTEC strains isolated from pigs. $8^{\text {th }}$ International Symposium on Shiga Toxin (Verocytotoxin) Producing Escherichia coli Infections (VTEC 2012). Zoonoses and Public Health 59:19-90.

Corogeanu D, Willmes R, Wolke Met al. (2012) Therapeutic concentrations of antibiotics inhibit Shiga toxin release from enterohemorrhagic E. coli O104:H4 from the 2011 German outbreak. BMC Microbiol 12:160.

Cromwell GL, Davis GW, Morrow WE et al. (1996) Efficacy of the antimicrobial compound U-82, 127 as a growth promoter for growing-finishing pigs. J Anim Sci 74:1284-1287.

Di Conza JA, Gutkind GO (2010) Integrones: los coleccionistas de genes. Rev Arg Microbiol 42:63-78.

Etcheverría AI, Padola NL (2013) Shiga toxin-producing Escherichia coli Factors involved in virulence and cattle colonization. Virulence 5:1-7.

Farina C, Goglio A, Conedera G et al. (1996) Antimicrobial susceptibility of Escherichia coli $\mathrm{O} 157$ and other enterohaemorrhagic Escherichia coli isolated in Italy. Eur J Clin Microbiol Infect Dis 15:351-353. 
Fluit AC, Schmitz FJ (1999) Class 1 Integrons, Gene Cassettes, Mobility and Epidemiology. Eur J Clin Microbiol Infect Dis 18:761-770.

Frank C, Werber D, Cramer JP (2011) Epidemic profile of Shigatoxin-producing Escherichia coli O104:H4 outbreak in Germany. N Engl J Med 365:1771-1780.

Guerra B, Junker E, Schroeter A et al. (2006) Phenotypic and genotypic characterization of antimicrobial resistance in Escherichia coli $\mathrm{O} 111$ isolates. J Antimicrob Chemother 57:1210-1214.

Hall RM, Collis CM (1995) Mobile gene cassettes and integrons: capture and spread of genes by site-specific recombination. Mol Microbiol 15:593-600.

Hansson K, Sundström L, Pelletier A et al. (2002) IntI2 Integron integrase in Tn7. J Bacteriol 184:1712-1721.

Hussein H, Sakuma T (2005) Prevalence of Shiga toxinproducing Escherichia coli in dairy cattle and their products. J Dairy Sci 88:450-465.

Ishii Y, Kimura S, Alba J et al. (2005) Extended-spectrum betalactamase-producing Shiga toxin gene (Stx1)-positive Escherichia coli $\mathrm{O} 26: \mathrm{H} 11$ : a new concern. J Clin Microbiol 43:1072-1075.

Jay MT, Cooley M, Carychao D et al. (2007) Escherichia coli O157:H7 in feral swine near spinach fields and cattle, central California coast. Emerg Infect Dis 13:1908-11.

Kaper JB, Nataro JP, Mobley HLT (2004) Pathogenic Escherichia coli. Nat. Rev. Microbiol 2:123-140.

La Ragione RM, Best A, Woodward MJ et al. (2009) Escherichia coli $\mathrm{O} 157: \mathrm{H} 7$ colonization in small domestic ruminants. FEMS Microbiol Rev 33:394-410.

Magiorakos AP, Srinivasan A, Carey RB et al. (2012) Multidrugresistant, extensively drug-resistant and pandrug-resistant bacteria: an international expert proposal for interim standard definitions for acquired resistance. Clin Microbiol Infect 8:268-281.

Mazel D (2006) Integrons: agents of bacterial evolution. Nat Rev Microbiol. 4:608-620.

McEwen SA, Fedorka-Cray PJ (2002) Antimicrobial use and resistance in animals. Clin Infect Dis 34:93-106.

Murinda SE, Ebner PD, Nguyen LT et al. (2005) Antimicrobial resistance and class 1 integrons in pathogenic Escherichia coli from dairy farms. Foodborne Pathog Dis 2:348-352.

Naylor SW, Gally DL, Low JC (2005) Enterohaemorrhagic E. coli in veterinary medicine. Int $\mathrm{J}$ Med Microbiol 295:419-441.

Newell D, Koopmans M, Verhoef L et al. (2010) Food-borne diseases - The challenges of 20 years ago still persist while new ones continue to emerge. J Food Microbiol 139:3-15.

Notario R, Fain Binda JC, Prado JV et al. (2000) Prevalencia de Escherichia coli Enterohemorrágico en una zona ganadera de Argentina. Caracterización genotípica de las cepas de origen animal. Rev Med Chile 128:1335-1341.

Parma AE, Sanz ME, Blanco JE et al. (2000) Virulence genotypes and serotypes of verotoxigenic Escherichia coli isolated from cattle and foods in Argentina. Importance in public health. Eur J Epidemiol 16:757-762.

Paton AW, Paton J (1998) Detection and characterization of Shiga Toxigenic Escherichia coli by using Multiplex PCR assays for $s t x_{1}, s_{x}$, eaeA, Enterohemorrhagic E. coli hlyA, $\mathrm{rfb}_{\mathrm{O} 111}$, and $\mathrm{rfb}_{\mathrm{O} 157}$. J Clin Microbiol 36:598-602.

Ploy MC, Francois D, Courvalin P et al. (2000a) Molecular characterization of Integrons in Acinetobacter baumannii: description of a Hybrid Class 2 Integron. Ant Agents Chemot 44:2684-2688.

Ploy MC, Lambert T, Couty JP et al. (2000b) Integrons: an antibiotic resistance gene capture and expression system. Clin Chem Lab Med 38:483-487.

Recchia GD, Hall RM (1995) Gene cassettes: a new class of mobile element. Rev Art Microbiol 141:3015-3027.

Singh R, Schroeder CM, Meng J et al. (2005) Identification of antimicrobial resistance and class 1 integrons in Shiga toxin-producing Escherichia coli recovered from humans and food animals. J Antimicrob Chemother 56:216-219.

Stokes HW, Hall RM (1989) A novel family of potentially mobile DNA elements encoding site-specific gene-integration functions: integrons. Mol Microbiol 3:1669-1683.

Thomas KM, McCann MS, Collery MM et al. (2012) Tracking verocytotoxigenic Escherichia coli O157, O26, O111, O103 and O145 in Irish cattle. Int J of Food Microb 153:288-296.

Valat C, Haenni M, Saras E et al. (2012) CTX-M-15 extendedspectrum beta-lactamase in a Shiga toxin producing Escherichia coli isolate of serotype O111:H8. Appl Environ Microbiol 78:1308-1309.

Vali L, Hamouda A, Hoyle DV et al. (2007) Antibiotic resistance and molecular epidemiology of Escherichia coli O26, O103 and $\mathrm{O} 145$ shed by two cohorts of Scottish beef cattle. J Antimicrob Chemother 59:403-410.

Vignoli R, Varela G, Mota MI et al. (2005) Enteropathogenic Escherichia coli strains carrying genes encoding the PER-2 and TEM-116 Extended-Spectrum Lactamases isolated from children with diarrhea in Uruguay. J Clinical Microbiol 43:2940-2943.

Voyer LE (1996) Síndrome Urémico Hemolítico. López Libreros Editores, Buenos Aires, pp 17-31.

Waldor M (2010) Mobilizable genomic islands: going mobile with oriT mimicry. Mol Microbiol 78:537-540.

Wetzel AN, LeJeune JT (2006) Clonal dissemination of Escherichia coli $\mathrm{O} 157: \mathrm{H} 7$ subtypes among dairy farms in northeast Ohio. Appl Environ Microbiol 72:2621-2626.

Zhao S, White DG, Beilei GE et al. (2001) Identification and characterization of integron-mediated antibiotic resistance among Shiga Toxin-Producing Escherichia coli isolates. Appl. Environ. Microbiol 4:1558-1564.

\section{]Associate Editor: Elaine Cristina Pereira De Martinis}

All the content of the journal, except where otherwise noted, is licensed under a Creative Commons License CC BY-NC. 